\title{
Towards 6G Network Slicing
}

\author{
Rodrigo Moreira ${ }^{1,2}$, Flávio de Oliveira Silva ${ }^{1}$ \\ ${ }^{1}$ Faculty of Computing (FACOM) \\ Federal University of Uberlândia (UFU), Uberlândia, MG, Brazil \\ ${ }^{2}$ Institute of Exact and Technological Sciences (IEP) \\ Federal University of Viçosa (UFV), Rio Paranaíba, MG, Brazil \\ \{rodrigo.moreira, flavio\}@ufu.br, rodrigo@ufv.br
}

\begin{abstract}
Networks should connect communicating peers, supporting vertical services requirements. The network evolution towards $6 G$ requires native network slicing techniques. Some literature approaches claim network slice realization, but they do not convincingly address the deployment across multiple Autonomous Systems. This work investigates the current $6 G$ network slicing landscape, presents some gaps, and introduces the concept of the recursive network slicing between multiple Autonomous Systems, supported by the NASOR approach. This innovative concept supports implementing new network services required by the $6 G$ vision. This work also sheds light on the $6 G$ requirements for network slicing.
\end{abstract}

\section{Introduction}

The purpose of networks is to connect communicating peers and provide their communication requirements. Networks' evolution requires their capacity to support different requirements of the top a common substrate.

5G highlighted this by defining three generic services called Enhanced Mobile Broadband (eMBB), Massive Machine-type Communications (mMTCs), and UltraReliable Low-Latency Communications (URLLCs) [Popovski et al. 2018] to support not only user-centric communication but also different business verticals. The trend of network specialization will continue and will require improvements in $6 \mathrm{G}$ [Dogra et al. 2020].

A Network Slice [NGMN Alliance 2016] is a logical network with specific characteristics, policies, and configurations. The network slice represents an on-demand, end-to-end logical network that runs on common hardware [Ordonez-Lucena et al. 2017] that supports user services and applications. The concept of network slicing is crucial to realize $6 \mathrm{G}$ vision [Chowdhury et al. 2020].

This work investigates the current $6 \mathrm{G}$ network slicing landscape, presenting some gaps that need to be fulfilled by network sling techniques to support the $6 \mathrm{G}$ vision. We also propose the concept of a recursive multi-domain network slice supported by the Network And Slice ORcrhestrator (NASOR) approach for network slicing between multiple Autonomous Systems (AS).

The remaining of this work is organized as follows: Section 2 presents an overview of the network slicing landscape regarding 6G. Section 3 describes our proposal of a recursive multi-domain network slicing approach that couples to the $3 \mathrm{GPP}$ management 
system and represents an innovative concept of network slicing to support $6 \mathrm{G}$ requirements. Finally, in Section 4 we draw some conclusions and present a future research agenda.

\section{The 6G Network Slicing Landscape}

[Maksymyuk et al. 2020] proposes a vision for a 6G architecture based on blockchain with support for distributed and virtualized computing and communication for a decentralized multi-operator $6 \mathrm{G}$ environment. The proposal covers the actors and transactions related to a dynamic infrastructure and an electromagnetic spectrum market. This vision does not detail the technological components that enable network slicing. $6 \mathrm{G}$ architecture envisions the ability to deploy network slices driven by application needs and adaptable to specific markets [Taleb et al. 2020].

[Samdanis and Taleb 2020] presents an architectural vision considering the advances of softwarization, agile control, and deterministic services over the $6 \mathrm{G}$ architecture. In addition, an architecture with modular components is envisaged to improve the customization and performance of network slicing.

In contrast to [Samdanis and Taleb 2020], we argue that the realization of deterministic services, customization of network parameters at the transport level is achievable through what we call recursive slicing, presented in Section 3.

A proposed paradigm shift named Mandate-driven Networking is presented by [Moerman et al. 2020]. To realize this paradigm, a hyperstrator, combines several network segments and administrative domains to carry out End-to-End (E2E) communication between humans or devices. In this proposal, it is considered that specific domains have their own orchestrators and that the requirements for the deployment of the network slice are expressed through the Expressive Application Program Interface (xAPI).

Our view of network slicing goes beyond the one presented by [Moerman et al. 2020]. We envision network slicing as a fundamental technological enabler for the $6 \mathrm{G}$ architecture, extending its concept to recursive network slicing across multiple domains. By enabling recursive network slicing, numerous user or market-specific applications are achievable, such as ManyNets [Taleb et al. 2020].

In [Ziegler et al. 2020] an architecture for the 6G era is discussed from the perspective of four aspects: platform, functions, orchestration, and specialization. Concerning specialization, 6G network architecture envisions offering specialized and performance attribute-oriented networks. This architectural block will contain mechanisms that perform extreme network slicing. Orchestration refers to components for offering open services, monetizing domain resources, and cognitive management and automation mechanisms.

The architectural vision of the 6G network proposed by [Ziegler et al. 2020], specifically the realization of network slicing, differs from our vision. We envision a $6 \mathrm{G}$ network architecture using a distributed-hierarchical orchestrator to perform recursive slicing. However, the recursive network slicing proposed here can be improved through the concept of deep slicing, allowing a fine adjustment of the various parameters that describe a slice.

[Räisänen 2020] presents a view of user requirements taking into account the evo- 
lution of 5G. In addition, the author points out his perception of the essential technological resources and enablers for the realization of $6 \mathrm{G}$

In [Räisänen 2020] it is considered that $6 \mathrm{G}$ network will provide dynamic network slicing. The 5G network has broadly addressed offering static and specialized connectivity as URLLC or eMBB. Our vision of network slicing expands the concept from dynamic to recursion. Thus, the owner of a network slice can dynamically resize it and offer subslices for specific applications and markets.

To summarize this brief landscape of network slicing for a $6 \mathrm{G}$ network, we propose Table 1. It presents characteristics such as Proposal Type, which refers to the type of contribution under analysis. Architecture Proposal contributions present components and technology enablers of a 6G network architecture. The Recursive Network Slicing feature denotes which work considers this concept. In this column, we distinguish our view of network slicing from other related work.

Other columns such as SDN, NFV, Blockchain and Artificial Intelligence are the technological enablers that the views or architectures consider essential to perform a $6 \mathrm{G}$ network slice. The column Multi-domain Orchestration characterizes if the solution envisions or considers the architectural block components that enable network slicing between multiple domains.

Table 1. Network Slicing Proposals Summary.

\begin{tabular}{|c|c|c|c|c|c|c|c|}
\hline Proposal & $\begin{array}{c}\text { Proposal } \\
\text { Type } \\
\end{array}$ & $\begin{array}{c}\text { Recursive } \\
\text { Network Slicing } \\
\end{array}$ & SDN & NFV & Blockchain & $\begin{array}{c}\text { Artificial } \\
\text { Intelligence }\end{array}$ & $\begin{array}{l}\text { Multi-domain } \\
\text { Orchestration } \\
\end{array}$ \\
\hline [Maksymyuk et al. 2020] & $\begin{array}{l}\text { Architectural } \\
\text { Proposal }\end{array}$ & $\mathrm{O}$ & 0 & $\mathrm{O}$ & 0 & ? & ? \\
\hline [Samdanis and Taleb 2020] & $\begin{array}{l}\text { Architectural } \\
\text { Vision }\end{array}$ & $\mathrm{O}$ & 0 & 0 & 0 & 0 & 0 \\
\hline $\begin{array}{c}\text { [Moerman et al. 2020] } \\
\text { [Ziegler et al. 2020] } \\
\text { [Räisänen 2020] }\end{array}$ & $\begin{array}{l}\text { Vision } \\
\text { Vision } \\
\text { Vision }\end{array}$ & $\begin{array}{l}0 \\
0 \\
0\end{array}$ & $\begin{array}{l}0 \\
0 \\
0\end{array}$ & 0 & $\begin{array}{l}0 \\
0 \\
0\end{array}$ & ? & ? \\
\hline Ours & $\begin{array}{l}\text { Architectural } \\
\text { Proposal }\end{array}$ & 0 & 0 & 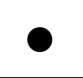 & 0 & O & 0 \\
\hline
\end{tabular}

In Table 1, we point out how some state-of-the-art approaches adopt these several concepts. The symbol (O) represents the complete adoption. The $(\boldsymbol{O})$ suggests that the paper partially adopts the concept, the $(\mathbf{O})$ means the concept is not mentioned in work.

\section{Recursive Multi-Domain Network Slicing}

Network slicing has received significant efforts from the scientific community, specifically in the mobile networks context [Foukas et al. 2017]. Recently, we proposed the NASOR approach for network slicing between multiple ASs [Moreira et al. 2020].

NASOR is an architectural framework that employs political and technological separation of domains through hierarchical-distributed orchestrators. These Orchestrators interact with each other asynchronously through a key-value repository. Also, they exchange domain capacity information and network slice deployment parameters. Through segment routing technology, NASOR identifies network slices along the data plane constructed by Internet routing algorithms.

There are many approaches in the state of the art which tried to realize network slicing. However, they predominantly focused on network slicing realization at mobile 


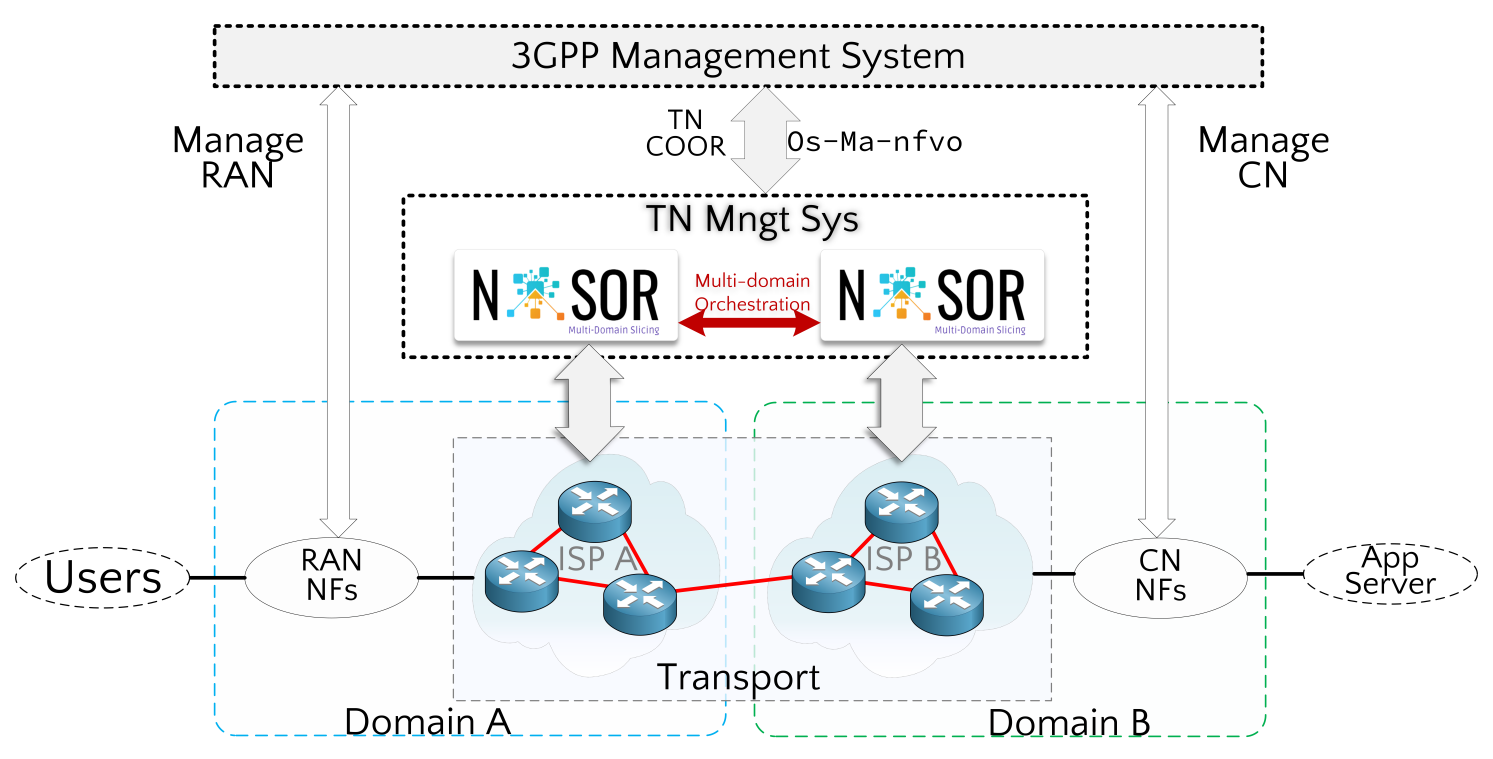

Figure 1. Recursive Network Slicing Proposal at the Transport Level.

network access. As illustrated in Figure. 1, we exploit network slicing to transport network level. A 3rd Generation Partnership Project (3GPP) leaves the transport level open to embed new technologies and management proposals and methods for network slicing realization at the transport level [3GPP 2018]. Hence, NASOR plays Network Slice Management Function (NSMF) roles to handle network slicing life-cycle on the transport level.

In this paper, we glimpse the 3GPP Management System interacting with the NASOR through the $O s-M a-n f v o$ interface to specify the network slice parameters. As the NASOR is hierarchical-distributed, the network slice deployment can span across multiple ASs. The NASOR builds a network slicing through the Network and Orchestration (NANO) component. NANO is the private orchestrator for a new network slicing. NANO enables the users or slice-owner to configure theirs network slicing according to the desired parameters, such as latency, throughput, or resize the network slice itself, creating new sub-slices. Rescaling a parent network slice by generating subslices of child networks is a recursive network slice.

Figure. 2 depicts how NASOR employs recursive network slicing for $6 \mathrm{G}$ networks. Upon receiving a network slice deployment request, NASOR instantiates a new NANO that will handle network slicing configuration and orchestration. NANO configures parameters across Internet routers to pave a data plane for the network slice. NANO sets up the Slice Forwarding Table, which contains the network slices identifiers. NANO handles Slice Metrics, defines Slice Capacity. According to Fig. 2, NANO enables slice-owner to create new slices, Slice 1 contains a Slice 2, which was recursively deployed. Eventually, Slice 2 may resize itself, creating a network subslice up to their parent network slicing resource limit.

The 6G architecture should enable more sophisticated use cases and meet stringent requirements such as latency and reliability. Thus, among the technological and conceptual enablers for performing network slicing, a recursive multi-domain network 


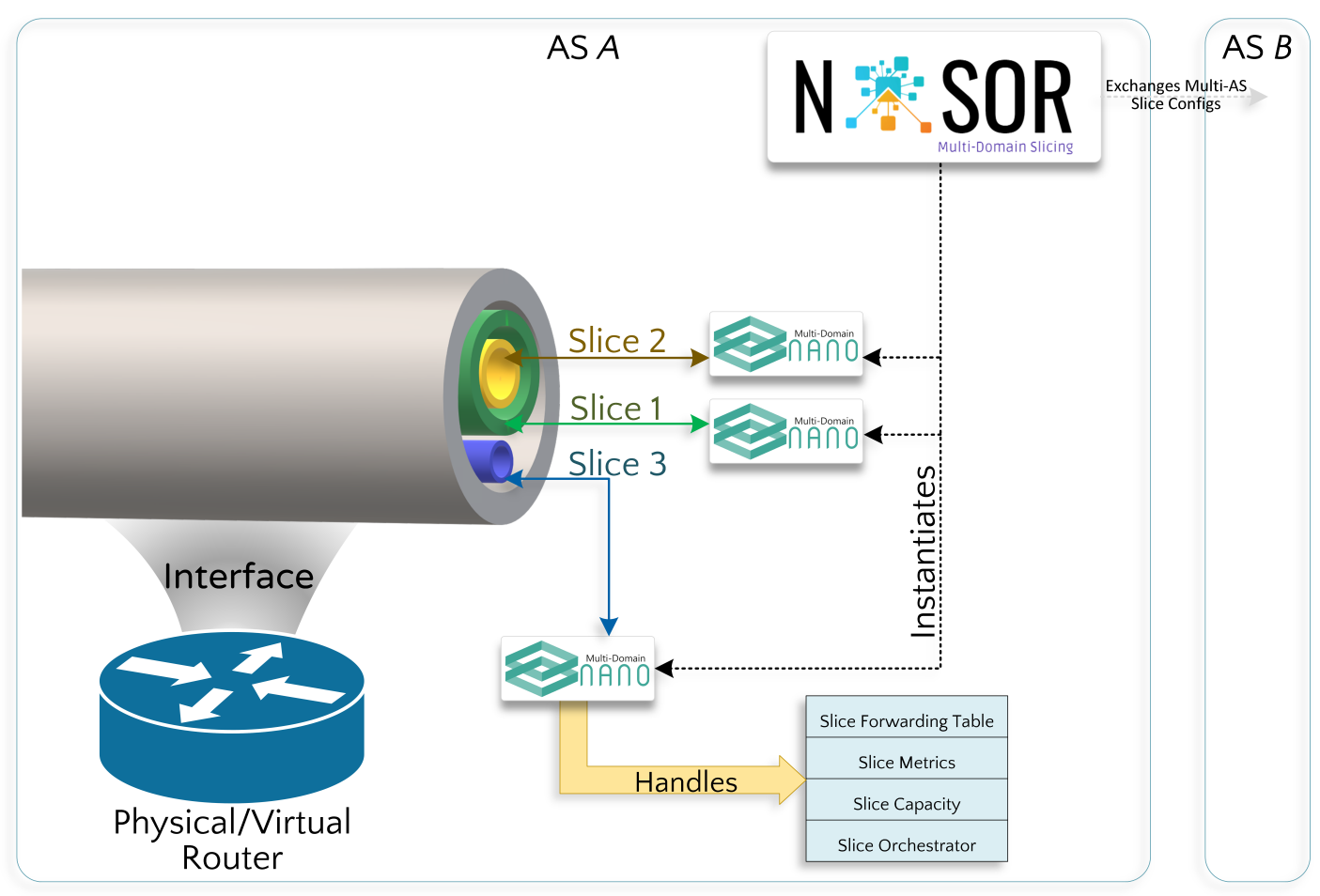

Figure 2. Recursive Multi-domain Network Slicing.

slicing mechanism emerged as a fundamental enabler. In addition, intelligent network slicing should shift paradigms with customized network slices that are adaptable to user demands.

\section{Concluding Remarks and Future Work}

Network slicing is critical to serving users in a personalized way. The one-size-fits-all concept makes innovative applications and business verticals over the network impossible. So, in this work, we present a brief landscape of network slicing regarding the $6 \mathrm{G}$ vision.

We also present NASOR, a framework for deploying network slices across multiple domains, and supports the concept of recursive network slicing. NASOR is a subsystem that interacts with the 3GPP Management System to perform recursive slicing at the transport level. Furthermore, we pointed out how the recursive slicing, enabled by NA$\mathrm{SOR}$, is an innovative concept to support the implementation of new network services required by the $6 \mathrm{G}$ vision.

In our research and development agenda, we intend to evaluate the recursive network slicing quantitatively, considering scalability, security, and isolation challenges. Furthermore, it seems critical to assess how artificial intelligence techniques can expand the possibilities regarding managing and intelligently orchestrating self-sustained recursive network slices considering, among others, smart slicing provisioning and performance assurance awareness.

\section{Acknowledgement}

This study was financed in part by the Coordenação de Aperfeiçoamento de Pessoal de Nível Superior - Brasil (CAPES) - Finance Code 001. 


\section{Referências}

3GPP (2018). 5G; management and orchestration; concepts, use cases and requirements (3GPP TS 28.530 version 15.0.0 Release 15).

Chowdhury, M. Z., Shahjalal, M., Ahmed, S., and Jang, Y. M. (2020). 6G Wireless Communication Systems: Applications, Requirements, Technologies, Challenges, and Research Directions. IEEE Open Journal of the Communications Society, 1:957-975. Conference Name: IEEE Open Journal of the Communications Society.

Dogra, A., Jha, R. K., and Jain, S. (2020). A Survey on beyond 5G network with the advent of 6G: Architecture and Emerging Technologies. IEEE Access, pages 1-1. Conference Name: IEEE Access.

Foukas, X., Patounas, G., Elmokashfi, A., and Marina, M. K. (2017). Network slicing in 5G: Survey and challenges. IEEE Communications Magazine, 55(5):94-100.

Maksymyuk, T., Gazda, J., Volosin, M., Bugar, G., Horvath, D., Klymash, M., and Dohler, M. (2020). Blockchain-empowered framework for decentralized network management in 6g. IEEE Communications Magazine, 58(9):86-92.

Moerman, I., Zeghlache, D., Shahid, A., Santos, J. F., DaSilva, L. A., David, K., Farscrotu, J., de Ridder, A., Liu, W., and Hoebeke, J. (2020). Mandate-driven networking eco-system: A paradigm shift in end-to-end communications. In 2020 2nd $6 G$ Wireless Summit (6G SUMMIT), pages 1-6, Levi, Finland. IEEE.

Moreira, R., Rosa, P. F., Aguiar, R. L., and de Oliveira Silva, F. (2020). Enabling multi-domain and end-to-end slice orchestration for virtualization everything functions (VxFs). In Advanced Information Networking and Applications, pages 830-844, Cham. Springer International Publishing.

NGMN Alliance (2016). Description of network slicing concept.

Ordonez-Lucena, J., Ameigeiras, P., Lopez, D., Ramos-Munoz, J. J., Lorca, J., and Folgueira, J. (2017). Network slicing for 5G with SDN/NFV: Concepts, architectures, and challenges. IEEE Communications Magazine, 55(5):80-87.

Popovski, P., Trillingsgaard, K. F., Simeone, O., and Durisi, G. (2018). 5G Wireless Network Slicing for eMBB, URLLC, and mMTC: A Communication-Theoretic View. IEEE Access, 6:55765-55779. Conference Name: IEEE Access.

Räisänen, V. (2020). A framework for capability provisioning in B5G. In 2020 2nd 6G Wireless Summit (6G SUMMIT), pages 1-4, Levi, Finland. IEEE.

Samdanis, K. and Taleb, T. (2020). The road beyond 5G: A vision and insight of the key technologies. IEEE Network, 34(2):135-141.

Taleb, T., Aguiar, R. L., Grida Ben Yahia, I., Chatras, B., Christensen, G., Chunduri, U., Clemm, A., Costa, X., Dong, L., Elmirghani, J., et al. (2020). White paper on $6 \mathrm{~g}$ networking.

Ziegler, V., Viswanathan, H., Flinck, H., Hoffmann, M., Räisänen, V., and Hätönen, K. (2020). 6G architecture to connect the worlds. IEEE Access, 8:173508-173520. 